\title{
Five Moments for Hand Hygiene: a focused, targeted approach to patient safety
}

\author{
Julie Storr \\ World Health Organization, Geneva, Switzerland
}

doi: 10.3396/ijic.V5i2.019.09

\begin{abstract}
Introduction
The Five Moments for Hand Hygiene approach has the potential to revolutionize infection control education and practice in relation to hand hygiene improvement. ${ }^{1}$ This simple approach forms a core tenet of the WHO Hand Hygiene Improvement Strategy, derived from the WHO Guidelines on Hand Hygiene in Health Care (Advanced Draft) ${ }^{2}$ and is an important part of the weaponry of tools available. It has been tested and applied in a range of settings and cultures, in both developed and developing countries. Infection control and prevention practitioners therefore face the question: what can the Five Moments approach do to improve patient safety within their organization? The answer will most likely depend on the maturity of the infection control programme, the position currently occupied on the continuum of hand hygiene improvement, existing infrastructures and the desire for new innovative approaches to enhance hand hygiene improvement strategies for long term success.
\end{abstract}

\section{Background}

The aim of Your Five Moments for Hand Hygiene is to streamline and simplify the number of times hand hygiene needs to occur within health care. It is concerned with promoting hand hygiene only at those moments within care and treatment sequences which are likely to yield the maximum return in terms of patient safety, and reduce the burden of unnecessary hand hygiene. Time to perform hand hygiene has been described as one of the key factors in persistently low compliance. ${ }^{3}$ Embracing the Five Moments approach positions hand hygiene as an integral part of the treatment/care process and attempts to eliminate doubt in terms of the required times when it should be applied. Implementation of the approach is concerned with enhancing reliability in terms of hand hygiene compliance.

The rationale for a continued focus on hand hygiene can be found in the results of recent baseline levels of compliance within both developed and developing countries which suggest that rates at baseline remain sub-optimal. ${ }^{4-5}$ Despite significant progress during recent decades, across all corners of the world, sustained hand hygiene improvements remain a significant challenge.

The reasons behind low compliance are multiple and differ depending on the context. At one end of the spectrum, healthcare facilities face huge problems in ensuring a satisfactory infrastructure, while in more developed countries infrastructures are increasingly already strengthened and the emphasis logically shifts to other components of the multimodal improvement strategy. Irrespective of this, infection control leaders including nurses and doctors, play a crucial role in health care system strengthening per se. The Five 
Moments approach can be central to this and is increasingly forming the bedrock of integrated infection control strategies of the modern age.

That hand hygiene improvement still warrants international attention has been illustrated by the overwhelming response to the call to action to $\mathrm{WHO}$ Member States by the First Global Patient Safety Challenge, Clean Care is Safer Care. This has resulted in political pledges of commitment being made by 116 ministries of health. Political commitment to take action on this recalcitrant problem has already translated to action at the "bedside" in a growing number of countries.

\section{Overview of the approach}

Five Moments is essentially a tool to position hand hygiene as an innovative, ergonomic, sought after and valued activity which is at the heart of professional performance. From a promotional perspective, hand hygiene becomes an action that is central to all patient areas/treatment procedures, rather than falling outside of this. Its strong visual imagery is intended to be widely displayed in clinical settings for the attention of managers, health care workers, patients and the public. It is predicated on the assumption that better hand hygiene compliance reduces pathogen transmission and contributes significantly to reducing health careassociated infection (HAI).

\section{Targeted and focused}

The approach targets the precise moments in time at which, if hand hygiene occurs, microbial transmission will be halted and future harm prevented. It is based on an appreciation of the high presence of microbes in specific geographical locations surrounding the patient in any health care context. The immediate geographical area (patient zone) surrounding a patient, i.e. the point of care, becomes the target of preventative action. The patient zone therefore includes the patient, their intact skin and his/her immediate surroundings colonized by patient flora. Beyond this, the environment is classified as the healthcare zone, which includes all other surfaces. The likelihood is that the healthcare zone is made-up of a mixture of microbes, from the multiple patients present within a hospital environment. Within the patient zone there are two critical times when hand hygiene must be performed; these relate to contact with 'clean sites' (Moment 2: Before Aseptic Task) and after contact with 'body fluid sites' (Moment 3: After Body Fluid Exposure Risk).

\section{Integral to the WHO Multimodal Strategy}

The WHO Multimodal Hand Hygiene Improvement Strategy, based on the WHO Guidelines on Hand hygiene in Healthcare, outlines the actions requires across a number of areas. The Five Moments approach is aligned to each of the five components of the strategy, as illustrated in the table below:

\begin{tabular}{lr} 
Multimodal component & $\begin{array}{r}\text { Potential } \\
\text { to address } \\
\text { via vehicle of } \\
\text { Five Moments }\end{array}$ \\
\hline System change & $\checkmark$ \\
\hline Training and education & $\checkmark$ \\
\hline Monitoring and evaluation & $\checkmark$ \\
\hline Reminders in the workplace & $\checkmark$ \\
\hline Safety culture & $\checkmark$ \\
\hline
\end{tabular}

It is clear therefore that as well as providing a visual reminder of the correct indications for hand hygiene, the approach is central to observational monitoring of compliance and subsequent feedback to clinical teams. Non-compliance with any of the moments results in the opportunity to target educational interventions. Observation of hand hygiene compliance based on the Five Moments provides a powerful symbol to healthcare workers and patients that time and effort on hand hygiene improvement is justified and that hand hygiene itself is a critical patient safety intervention

\section{Value-added}

Implementation of the Five Moments approach can trigger debate and discussion among health care teams and this dialogue should be welcomed as part of ongoing knowledge transfer concerning infection prevention and control. The approach can be used to instil a sense of ownership amongst clinical staff concerning the principles of germ transmission. WHO have produced a suite of accompanying educational resources to assist in observational compliance monitoring, centred around the Five Moments Concept: 
- A Manual for Observers

- A Training DVD - comprised of real-life simulated scenarios

- Training slides

- WHO Hand Hygiene Observation Form

- Your Five Moments for Hand Hygiene Posters

The Manual for the Observers explains how to use the tools and additional information is also included on the WHO Hand Hygiene Observation Form. The "Training film" with its 9 scenarios is designed to help convey the approach of the Five Moments for Hand Hygiene to observers and health-care workers. Included within the DVD is a demonstration of the correct technique for hand washing and handrubbing.

In summary, the Five Moments approach is designed to move healthcare towards a situation where compliance at these key Five Moments becomes a habitual, sub-conscious action. Your Five Moments for Hand Hygiene has universal applicability. It is an exciting innovation for infection control practitioners and has the potential to make hand hygiene improvement "sticky" and thus contribute significantly to sustainability and spread.

\section{Links}

Multimodal strategy:

http://www.who.int/gpsc/news/simple_guideline/en/index.html My Five Moments for hand hygiene:

http://www.who.int/gpsc/tools/Five_moments/en/index.html

\section{National campaigns:}

\http://www.who.int/gpsc/national_campaigns/en/ WHO World Alliance for Patient Safety homepage: http://www.who.int/patientsafety/en/

WHO Member States pledging commitment to Clean Care is Safer Care:

http://www.who.int/gpsc/media/Pledge_countriesOctober_08.pdf

\section{References}

1. Sax H, Allegranzi B, Uckay I, Larson E, Boyce J, Pittet D. 'My five moments for hand hygiene': a user-centred design approach to understand, train, monitor and report hand hygiene. Journal of Hospital Infection 2007; 67: 9-21.

2. World Health Organization. WHO Guidelines for Hand Hygiene in Health Care (Advanced Draft). Geneva: World Health Organization; 2006.

3. Voss A, Widmer AF. No time for handwashing!? Handwashing versus alcoholic rub: can we afford 100\% compliance? Infect Control and Hosp Epidemiol 1997; 18: 205-208.

4. Allegranzi B, Bengaly L, Minta DK, et al. on behalf of the World Health Organization (WHO) "Point $\mathrm{G}^{\text {" Project Management }}$ Committee. Assessing hand hygiene perception, compliance and structures in a university hospital in Mali. Geneva Forum: Towards Global Access to Health 2008, Geneva, Switzerland; May 25-28, 2008 (available from the World Alliance for Patient Safety).

5. Grayson ML, Jarvie LJ, Martin R, et al. Significant reductions in methicillin-resistant Staphylococcus aureus bacteraemia and clinical isolates associated with a multisite, hand hygiene culture-change program and subsequent successful statewide roll-out. Med J Aust 2008; 188: 633-640.

The author would like to make clear that the conference presentation on which this extended abstract is derived was delivered before the finalization of the WHO Guidelines on Hand Hygiene in Health Care, the final version being published on 5th May 2009. 